International Journal of Pure and Applied Mathematics

Volume 106 No. 2 2016, 611-624

ISSN: 1311-8080 (printed version); ISSN: 1314-3395 (on-line version)

url: http://www.ijpam.eu

doi: 10.12732/ijpam.v106i2.23

ijpam.eu

\title{
COMMON FIXED POINTS FOR SEMI-COMPATIBLE MAPPINGS IN MULTIPLICATIVE METRIC SPACES
}

\author{
Parveen Kumar ${ }^{1}$, Sanjay Kumar ${ }^{1}$, Shin Min Kang ${ }^{2} \S$ \\ ${ }^{1}$ Department of Mathematics \\ Deenbandhu Chhotu Ram University of Science and Technology \\ Murthal, Sonipat 131039, Haryana, INDIA \\ ${ }^{2}$ Department of Mathematics and RINS \\ Gyeongsang National University \\ Jinju 52828, KOREA
}

\begin{abstract}
In this paper, we introduce the notion of semi-compatible mappings in multiplicative metric spaces and establish common fixed point theorems for those mappings.
\end{abstract}

AMS Subject Classification: multiplicative metric spaces, semi-compatible mappings, weakly compatible mappings

Key Words: 47H10, 54H25

\section{Introduction and Preliminaries}

It is well know that the set of positive real numbers $\mathbb{R}_{+}$is not complete according to the usual metric. To overcome this problem, in 2008, Bashirov et al. [3] introduced the concept of multiplicative metric spaces as follows:

Received: November 23, 2015

Published: February 18, 2016

$\S_{\text {Correspondence author }}$
(C) 2016 Academic Publications, Ltd. url: www.acadpubl.eu 
Definition 1.1. Let $X$ be a nonempty set. A multiplicative metric is a mapping $d: X \times X \rightarrow \mathbb{R}_{+}$satisfying the following conditions:

(i) $d(x, y) \geq 1$ for all $x, y \in X$ and $d(x, y)=1$ if and only if $x=y$;

(ii) $d(x, y)=d(y, x)$ for all $x, y \in X$;

(iii) $d(x, y) \leq d(x, z) \cdot d(z, y)$ for all $x, y, z \in X$ (multiplicative triangle inequality).

Then the mapping $d$ together with $X$, that is, $(X, d)$ is a multiplicative metric space.

Example 1.2. ([10]) Let $\mathbb{R}_{+}^{n}$ be the collection of all $n$-tuples of positive real numbers. Let $d^{*}: \mathbb{R}_{+}^{n} \times \mathbb{R}_{+}^{n} \rightarrow \mathbb{R}$ be defined as follows:

$$
d^{*}(x, y)=\left|\frac{x_{1}}{y_{1}}\right|^{*} \cdot\left|\frac{x_{2}}{y_{2}}\right|^{*} \cdots\left|\frac{x_{n}}{y_{n}}\right|^{*},
$$

where $x=\left(x_{1}, \ldots, x_{n}\right), y=\left(y_{1}, \ldots, y_{n}\right) \in \mathbb{R}_{+}^{n}$ and $|\cdot|^{*}: \mathbb{R}_{+} \rightarrow \mathbb{R}_{+}$is defined by

$$
|a|^{*}= \begin{cases}a & \text { if } a \geq 1 \\ \frac{1}{a} & \text { if } a<1 .\end{cases}
$$

Then it is obvious that all conditions of a multiplicative metric are satisfied. Therefore $\left(\mathbb{R}_{+}^{n}, d^{*}\right)$ is a multiplicative metric space.

Example 1.3. ([12]) Let $d: \mathbb{R} \times \mathbb{R} \rightarrow[1, \infty)$ be defined as $d(x, y)=a^{|x-y|}$, where $x, y \in \mathbb{R}$ and $a>1$. Then $d$ is a multiplicative metric and $(\mathbb{R}, d)$ is a multiplicative metric space. We may call it usual multiplicative metric spaces.

Remark 1.4. We note that the Example 1.2 is valid for positive real numbers and Example 1.3 is valid for all real numbers.

Example 1.5. ([12]) Let $(X, d)$ be a metric space. Define a mapping $d_{a}$ on $X$ by

$$
d_{a}(x, y)=a^{d(x, y)}= \begin{cases}1 & \text { if } x=y \\ a & \text { if } x \neq y\end{cases}
$$

where $x, y \in X$ and $a>1$. Then $d_{a}$ is a multiplicative metric and $\left(X, d_{a}\right)$ is known as the discrete multiplicative metric space.

Example 1.6. ([1]) Let $X=C^{*}[a, b]$ be the collection of all real-valued multiplicative continuous functions on $[a, b] \subset \mathbb{R}_{+}$. Then $(X, d)$ is a multiplicative metric space with $d$ defined by $d(f, g)=\sup _{x \in[a, b]}\left|\frac{f(x)}{g(x)}\right|$ for arbitrary $f, g \in X$. 
Remark 1.7. ([12]) We note that multiplicative metrics and metric spaces are independent.

Indeed, the mapping $d^{*}$ defined in Example 1.2 is multiplicative metric but not metric as it does not satisfy triangular inequality. Consider

$$
d^{*}\left(\frac{1}{3}, \frac{1}{2}\right)+d^{*}\left(\frac{1}{2}, 3\right)=\frac{3}{2}+6=7.5<9=d^{*}\left(\frac{1}{3}, 3\right) .
$$

On the other hand the usual metric on $\mathbb{R}$ is not multiplicative metric as it doesnt satisfy multiplicative triangular inequality, since

$$
d(2,3) \cdot d(3,6)=3<4=d(2,6) .
$$

One can refer to $[7,10]$ for detailed multiplicative metric topology.

Definition 1.8. Let $(X, d)$ be a multiplicative metric space. Then a sequence $\left\{x_{n}\right\}$ in $X$ said to be

(1) a multiplicative convergent to $x$ if for every multiplicative open ball $B_{\epsilon}(x)=\{y \mid d(x, y)<\epsilon\}, \epsilon>1$, there exists $N \in \mathbb{N}$ such that $x_{n} \in B_{\epsilon}(x)$ for all $n \geq N$, that is, $d\left(x_{n}, x\right) \rightarrow 1$ as $n \rightarrow \infty$.

(2) a multiplicative Cauchy sequence if for all $\epsilon>1$, there exists $N \in \mathbb{N}$ such that $d\left(x_{n}, x_{m}\right)<\epsilon$ for all $m, n \geq N$, that is, $d\left(x_{n}, x_{m}\right) \rightarrow 1$ as $n, m \rightarrow \infty$.

(3) We call a multiplicative metric space complete if every multiplicative Cauchy sequence in it is multiplicative convergent to $x \in X$.

Remark 1.9. The set of positive real numbers $\mathbb{R}_{+}$is not complete according to the usual metric. Let $X=\mathbb{R}_{+}$and the sequence $\left\{x_{n}\right\}=\left\{\frac{1}{n}\right\}$. It is obvious $\left\{x_{n}\right\}$ is a Cauchy sequence in $X$ with respect to usual metric and $X$ is not a complete metric space, since $0 \notin \mathbb{R}_{+}$. In case of a multiplicative metric space, we take a sequence $\left\{x_{n}\right\}=\left\{a^{\frac{1}{n}}\right\}$, where $a>1$. Then $\left\{x_{n}\right\}$ is a multiplicative Cauchy sequence since for $n \geq m$,

$$
\begin{aligned}
d\left(x_{n}, x_{m}\right) & =\left|\frac{x_{n}}{x_{m}}\right|=\left|\frac{a^{\frac{1}{n}}}{a^{\frac{1}{m}}}\right|=\left|a^{\frac{1}{n}-\frac{1}{m}}\right| \\
& \leq a^{\frac{1}{m}-\frac{1}{n}}<a^{\frac{1}{m}}<\epsilon \text { if } m>\frac{\log a}{\log \epsilon}
\end{aligned}
$$

where $|a|=\left\{\begin{array}{ll}a & \text { if } a \geq 1, \\ \frac{1}{a} & \text { if } a<1 .\end{array}\right.$ Also, $\left\{x_{n}\right\} \rightarrow 1$ as $n \rightarrow \infty$ and $1 \in \mathbb{R}_{+}$. Hence $(X, d)$ is a complete multiplicative metric space.

In 2012, Özavsar and Çevikel [10] gave the concept of multiplicative contraction mappings and proved some fixed point theorem of such mappings in a multiplicative metric space. 
Definition 1.10. Let $f$ be a mapping of a multiplicative metric space $(X, d)$ into itself. Then $f$ is said to be a multiplicative contraction if there exists a real number $\lambda \in[0,1)$ such that

$$
d(f x, f y) \leq d^{\lambda}(x, y) \text { for all } x, y \in X .
$$

In 2015, Kang et al. [9] introduced the concept of compatible mappings in multiplicative metric spaces as follows:

Definition 1.11. Let $f$ and $g$ be mappings of a multiplicative metric space $(X, d)$ into itself. Then $f$ and $g$ are said to be compatible if $\lim _{n \rightarrow \infty} d\left(f g x_{n}, g f x_{n}\right)=$ 1 , whenever $\left\{x_{n}\right\}$ is a sequence in $X$ such that $\lim _{n \rightarrow \infty} f x_{n}=\lim _{n \rightarrow \infty} g x_{n}=t$ for some $t \in X$.

In 1996, Jungck [8] introduced the concept of weakly compatible mappings and proved fixed point theorems using these mappings in metric spaces (see $[2,5,6,11])$.

Now, we introduce the notion in multiplicative metric spaces.

Definition 1.12. Let $f$ and $g$ be mappings of a multiplicative metric space $(X, d)$ into itself. Then $f$ and $g$ are said to be weakly compatible if they commute at coincidence points, that is, if $f t=g t$ for some $t \in X$ implies $f g t=g f t$.

In 1995, Cho et al. [4] introduced the concept of semi-compatibility in topological spaces.

Let $f$ and $g$ be mappings of a topological space into itself. Then $f$ and $g$ are said to be semi-compatible if

(1) $f y=g y$ implies $f g y=g f y$ and

(2) $\left\{f x_{n}\right\} \rightarrow u$ and $\left\{g x_{n}\right\} \rightarrow u$ imply $f g x_{n} \rightarrow g u$ as $n \rightarrow \infty$.

However, (2) implies (1) taking $x_{n}=y$ and $u=g y=f y$.

Now we define the semi-compatibility by the condition (2) only in the setting of a multiplicative metric space as follow:

Definition 1.13. Let $f$ and $g$ be mappings of a multiplicative metric space $(X, d)$ into itself. Then $f$ and $g$ are said to be semi-compatible if $\lim _{n \rightarrow \infty} d\left(f g x_{n}, g u\right)=1$, whenever $\left\{x_{n}\right\}$ is a sequence in $X$ such that $\lim _{n \rightarrow \infty} f x_{n}=$ $\lim _{n \rightarrow \infty} g x_{n}=u$ for some $u \in X$.

It follows that $f$ and $g$ are semi-compatible and $f y=g y$ imply $f g y=g f y$.

We note that $f$ and $g$ are semi-compatible, but it need not be compatible. Further it is shown that semi-compatiblity of $f$ and $g$ does not imply semicompatible of $g$ and $f$. 
Example 1.14. Let $X=[1,3]$ and $d: X \times X \rightarrow[1, \infty)$ be defined as $d(x, y)=a^{|x-y|}$, where $x, y \in X$ and $a>1$. Then $(X, d)$ is a multiplicative metric space. Define mappings $f, g: X \rightarrow X$ by

$$
f x=\left\{\begin{array}{ll}
x & \text { if } 1 \leq x<2, \\
3 & \text { if } 2 \leq x \leq 3,
\end{array} \quad g x= \begin{cases}4-x & \text { if } 1 \leq x<2, \\
3 & \text { if } 2 \leq x \leq 3\end{cases}\right.
$$

Consider $x_{n}=2-\frac{1}{n}$. Then $f x_{n}=2-\frac{1}{n}$ and $g x_{n}=2+\frac{1}{n}$ and so $f x_{n} \rightarrow 2$ and $g x_{n} \rightarrow 2=u$, say. Further,

$$
f g x_{n}=f\left(2+\frac{1}{n}\right)=3 \quad \text { and } \quad g f x_{n}=g\left(2-\frac{1}{n}\right)=2+\frac{1}{n} .
$$

Now

$$
\lim _{n \rightarrow \infty} d\left(f g x_{n}, g f x_{n}\right)=a^{|3-2|}=a \neq 1 .
$$

This implies that $f$ and $g$ are not compatible. Also,

$$
\lim _{n \rightarrow \infty} d\left(f g x_{n}, g u\right)=a^{|3-3|}=1 .
$$

So, $f$ and $g$ are semi-compatible and

$$
\lim _{n \rightarrow \infty} d\left(g f x_{n}, f u\right)=a^{|2-3|}=a \neq 1 .
$$

So, $g$ and $f$ are not semi-compatible.

Next, we show that semi-compatible is weakly compatible. For any $x \in$ $[1,2)$, it is obvious. Also for any $x \in[2,3], f x=g x=3$ and $f g x=f 3=3$, $g f x=g 3=3$. Thus $f$ and $g$ are weakly compatible.

Example 1.15. Let $X=[0,1]$ and $d: X \times X \rightarrow[1, \infty)$ be defined as $d(x, y)=a^{|x-y|}$, where $x, y \in X$ and $a>1$. Then $(X, d)$ is a multiplicative metric space. Define mappings $f, g: X \rightarrow X$ by

$$
f x=1-x, \quad g x= \begin{cases}1-x & \text { if } 0 \leq x<\frac{1}{3} \\ \frac{2}{3} & \text { if } x=\frac{1}{3}, \\ 1 & \text { if }\left(\frac{1}{3}, 1\right)-\frac{2}{3} \\ \frac{1}{3} & \text { if } x=\frac{2}{3} .\end{cases}
$$

Consider $x_{n}=\frac{1}{3}-\frac{1}{n}$. Then $f x_{n}=\frac{2}{3}+\frac{1}{n}$ and $g x_{n}=\frac{2}{3}+\frac{1}{n}$ and so $f x_{n}, g x_{n} \rightarrow$ $\frac{2}{3}=u$, say. Now,

$$
f g x_{n}=f\left(\frac{2}{3}+\frac{1}{n}\right)=\frac{1}{3}-\frac{1}{n} \quad \text { and } \quad g f x_{n}=g\left(\frac{2}{3}+\frac{1}{n}\right)=1 .
$$


This implies that $f$ and $g$ are not compatible. Further

$$
\lim _{n \rightarrow \infty} d\left(f g x_{n}, g u\right)=a^{\left|\frac{1}{3}-\frac{1}{3}\right|}=1 \quad \text { and } \quad \lim _{n \rightarrow \infty} d\left(g f x_{n}, f u\right)=a^{\left|1-\frac{1}{3}\right|} \neq 1 .
$$

So, $f$ and $g$ are semi-compatible, but $g$ and $f$ are not semi-compatible.

Weak compatibility does not imply semi-compatibility. Here $g$ and $f$ are weakly compatible as they commute at their coincidence point $\frac{2}{3}$, but the pair is not semi-compatible. Semi-compatiblity does not necessary imply compatiblity as $\lim _{n \rightarrow \infty} d\left(f g x_{n}, g f x_{n}\right) \neq 1$ in Examples 1.14 and 1.15 .

In next example we show that compatible does not necessary imply semicompatible.

Example 1.16. Let $X=[0,1]$ and $d: X \times X \rightarrow[1, \infty)$ be defined as $d(x, y)=a^{|x-y|}$, where $x, y \in X$ and $a>1$. Then $(X, d)$ is a multiplicative metric space. Define mappings $f, g: X \rightarrow X$ by

$$
f x=x, \quad g x= \begin{cases}x & \text { if } 0 \leq x<\frac{1}{3} \\ \frac{1}{2} & \text { if } x=\frac{1}{3} \\ \frac{1}{2} & \text { if } x>\frac{1}{3}\end{cases}
$$

Consider $x_{n}=\frac{1}{3}-\frac{1}{n}$. Then $f x_{n}=\frac{1}{3}-\frac{1}{n}$ and $g x_{n}=\frac{1}{3}-\frac{1}{n}$ and hence $f x_{n}, g x_{n} \rightarrow \frac{1}{3}=u$, say. Also,

$$
f g x_{n}=\frac{1}{3}-\frac{1}{n} \rightarrow \frac{1}{3} \quad \text { and } \quad g f x_{n}=\frac{1}{3}-\frac{1}{n} \rightarrow \frac{1}{3} .
$$

Further

$$
\lim _{n \rightarrow \infty} d\left(f g x_{n}, g f x_{n}\right)=1 .
$$

Hence $f$ and $g$ are compatible. But

$$
\lim _{n \rightarrow \infty} d\left(f g x_{n}, g u\right)=a^{\left|\frac{1}{3}-\frac{1}{2}\right|} \neq 1 .
$$

This imples that $f$ and $g$ are not semi-compatible.

\section{Main Results}

Now we give our main theorems. 
Theorem 2.1. Let $A, B, S$ and $T$ be mappings of a complete multiplicative metric space $(X, d)$ into itself satisfying the following:

$$
\begin{gathered}
A(X) \subset T(X) \text { and } B(X) \subset S(X) \\
d^{3}(A x, B y) \leq\left\{\operatorname { m a x } \left\{d^{3}(S x, T y), d^{3}(A x, S x), d^{3}(T y, B y),\right.\right. \\
\left.\left.d^{2}(T y, B y), d(S x, B y), d(A x, T y)\right\}\right\}^{\lambda}
\end{gathered}
$$

for all $x, y \in X$, where $\lambda \in(0,1)$;

$\left(C_{3}\right)$ either $A$ or $B$ is continuous;

$\left(C_{4}\right) A$ and $S$ are semi-compatible and $B$ and $T$ are weakly compatible.

Then $A, B, S$ and $T$ have a unique common fixed point in $X$.

Proof. Let $x_{0} \in X$ be any arbitrary point. Since $A(X) \subset T(X)$ and $B(X) \subset$ $S(X)$, there exists a point $x_{1} \in X$ such that $A x_{0}=T x_{1}$ and for this point $x_{1}$, there exists a point $x_{2} \in X$ such that $B x_{1}=S x_{2}$. Inductively, we can define a sequence $\left\{y_{n}\right\}$ in $X$ such that

$$
y_{2 n+1}=T x_{2 n+1}=A x_{2 n}, \quad y_{2 n}=S x_{2 n}=B x_{2 n-1}
$$

for $n=1,2, \ldots$.

On putting $x=x_{2 n}$ and $y=x_{2 n+1}$ in $\left(C_{2}\right)$, we have

$$
\begin{aligned}
& d^{3}\left(y_{2 n+1}, y_{2 n+2}\right) \\
&=d^{3}\left(A x_{2 n}, B x_{2 n+1}\right) \\
& \leq\{ \max \left\{\left(d^{3}\left(S x_{2 n}, T x_{2 n+1}\right), d^{3}\left(A x_{2 n}, S x_{2 n}\right), d^{3}\left(T x_{2 n+1}, B x_{2 n+1}\right),\right.\right. \\
&\left.\left.d^{2}\left(T x_{2 n+1}, B x_{2 n+1}\right), d\left(S x_{2 n}, B x_{2 n+1}\right), d\left(A x_{2 n}, T x_{2 n+1}\right)\right\}\right\}^{\lambda} \\
& \leq\left\{\operatorname { m a x } \left\{d^{3}\left(y_{2 n}, y_{2 n+1}\right), d^{3}\left(y_{2 n}, y_{2 n+1}\right), d^{3}\left(y_{2 n+1}, y_{2 n+2}\right),\right.\right. \\
&\left.\left.d^{2}\left(y_{2 n+1}, y_{2 n+2}\right), d\left(y_{2 n}, y_{2 n+2}\right), d\left(y_{2 n+1}, y_{2 n+1}\right)\right\}\right\}^{\lambda} \\
& \leq\left\{\max \left\{d^{3}\left(y_{2 n}, y_{2 n+1}\right), d^{3}\left(y_{2 n+2}, y_{2 n+1}\right)\right\}\right\}^{\lambda} .
\end{aligned}
$$

If $\max \left\{d^{3}\left(y_{2 n}, y_{2 n+1}\right), d^{3}\left(y_{2 n+1}, y_{2 n+2}\right)\right\}=d^{3}\left(y_{2 n+1}, y_{2 n+2}\right)$, which is a contradiction, therefore

$$
d^{3}\left(y_{2 n+1}, y_{2 n+2}\right) \leq d^{3 \lambda}\left(y_{2 n}, y_{2 n+1}\right)
$$

which implies that

$$
d\left(y_{2 n+1}, y_{2 n+2}\right) \leq d^{\lambda}\left(y_{2 n}, y_{2 n+1}\right)
$$


Similarly, we have

$$
d\left(y_{2 n}, y_{2 n+1}\right) \leq d^{\lambda}\left(y_{2 n-1}, y_{2 n}\right)
$$

It follow that

$$
\begin{aligned}
d\left(y_{n}, y_{n+1}\right) & \leq d^{\lambda}\left(y_{n-1}, y_{n}\right) \\
& \leq d^{\lambda^{2}}\left(y_{n-2}, y_{n-1}\right) \\
& \leq \cdots \leq d^{\lambda^{n}}\left(y_{0}, y_{1}\right)
\end{aligned}
$$

Let $m, n \in \mathbb{N}$ with $m>n$. Then

$$
\begin{aligned}
d\left(y_{n}, y_{m}\right) & \leq d\left(y_{n}, y_{n+1}\right) \cdot d\left(y_{n+1}, y_{n+2}\right) \cdots d\left(y_{m-1}, y_{m}\right) \\
& \leq d^{\lambda^{n}+\lambda^{n+1}+\cdots+\lambda^{m-1}}\left(y_{0}, y_{1}\right) \\
& \leq d^{\frac{\lambda^{n}}{1-\lambda}}\left(y_{0}, y_{1}\right) .
\end{aligned}
$$

This implies that $d\left(y_{m}, y_{n}\right) \rightarrow 1$ as $n \rightarrow \infty$. Hence $\left\{y_{n}\right\}$ is a multiplicative Cauchy sequence. Therefore, $y_{n} \rightarrow z \in X(N \rightarrow \infty)$. Thus its subsequences $A x_{2 n}, S x_{2 n}, B x_{2 n+1}$ and $T x_{2 n+1} \rightarrow z$.

Now, suppose that $A$ is continuous. Then $A A x_{2 n} \rightarrow A z$ and $A S x_{2 n} \rightarrow A z$. Since $A$ and $S$ are semi-compatible, $A S x_{2 n} \rightarrow S z$. By uniqueness of the limit in a multiplicative metric space, we obtain $A z=S z$. On putting $x=z$ and $y=x_{2 n+1}$ in $\left(C_{2}\right)$, we have

$$
\begin{aligned}
& d^{3}\left(A z, B x_{2 n+1}\right) \\
& \leq\left\{\operatorname { m a x } \left\{d^{3}\left(S z, T x_{2 n+1}\right), d^{3}(A z, S z), d^{3}\left(T x_{2 n+1}, B x_{2 n+1}\right)\right.\right. \\
& \left.\left.\quad d^{2}\left(T x_{2 n+1}, B x_{2 n+1}\right), d\left(S z, B x_{2 n+1}\right), d\left(A z, T x_{2 n+1}\right)\right\}\right\}^{\lambda} .
\end{aligned}
$$

By taking limit $n \rightarrow \infty$, we have

$$
\begin{gathered}
d^{3}(A z, z) \leq\left\{\operatorname { m a x } \left\{d^{3}(S z, z), d^{3}(A z, S z), d^{3}(z, z)\right.\right. \\
\left.\left.\left.d^{2}(z, z)\right), d(S z, z), d(A z, z)\right\}\right\}^{\lambda}
\end{gathered}
$$

that is,

$$
\left.d^{3}(A z, z)\right) \leq d^{3 \lambda}(A z, z)
$$

which implies that $A z=z$. Since $A(X) \subset T(X)$, there exists a point $v \in X$ such that $z=A z=T v$. 
By taking $x=z$ and $y=v$ in $\left(C_{2}\right)$, we have

$$
\begin{aligned}
d^{3}(z, B v)= & d^{3}(A z, B v) \\
\leq & \left\{\operatorname { m a x } \left\{d^{3}(S z, T v), d^{3}(A z, S z), d^{3}(T v, B v),\right.\right. \\
& \left.\left.d^{2}(T v, B v), d(S z, B v), d(A z, T v)\right\}\right\}^{\lambda} \\
= & \left\{\operatorname { m a x } \left\{d^{3}(S z, A z), d^{3}(A z, S z), d^{3}(z, B v),\right.\right. \\
& \left.\left.d^{2}(z, B v), d(S z, B v), d(A z, T v)\right\}\right\}^{\lambda} \\
= & \left\{\max \left\{1,1, d^{3}(z, B v), d^{2}(z, B v), d(z, B v), 1\right\}\right\}^{\lambda},
\end{aligned}
$$

which implies that $B z=z$. Hence $z=B v=T v$. Since $B$ and $T$ are weakly compatible, $T B v=B T v$. Hence $T z=B z$.

By taking $x=z$ and $y=z$ in $\left(C_{2}\right)$, we have

$$
\begin{aligned}
d^{3}(A z, B z) \leq\{ & \max \left\{d^{3}(S z, T z), d^{3}(A z, S z), d^{3}(T z, B z),\right. \\
& \left.\left.d^{2}(T z, B z), d(S z, B z), d(A z, T z)\right\}\right\}^{\lambda} \\
= & \left\{\max \left\{d^{3}(A z, B z), 1,1,1, d(A z, B z), d(A z, B z)\right\}\right\}^{\lambda},
\end{aligned}
$$

which implies that $A z=B z$. Hence $z=A z=B z=S z=T z$ and hence that is $z$ a common fixed point of $A, B, S$ and $T$.

Similarly, we can complete the proof when $B$ is continuous.

Finally in order to prove the uniqueness of $z$, let $w(w \neq z)$ be another common fixed point of $A, B, S$ and $T$. Then $w=A w=B w=S w=T w$.

By taking $x=z$ and $y=w$ in $\left(C_{2}\right)$, we have

$$
\begin{aligned}
d^{3}(z, w)= & d^{3}(A z, B w) \\
\leq & \left\{\operatorname { m a x } \left\{d^{3}(S z, T w), d^{3}(A z, S z), d^{3}(T w, B w),\right.\right. \\
& \left.\left.d^{2}(T w, B w), d(S z, B w), d(A z, T w)\right\}\right\} \\
= & \left\{\max \left\{d^{3}(z, w), 1,1,1, d(z, w), d(z, w)\right\}\right\}^{\lambda},
\end{aligned}
$$

which implies that $z=w$. Therefore $A, B, S$ and $T$ have a unique common fixed point in $X$. This complete the proof.

In Theorem 2.1, if we put $A=B$ and $S=T$, then we have following corollary.

Corollary 2.2. Let $A$ and $S$ be mappings of a complete multiplicative metric space $(X, d)$ into itself satisfying the following:

$$
A(X) \subset S(X) ;
$$


$\left(C_{6}\right)$

$$
\begin{gathered}
d^{3}(A x, A y) \leq\left\{\operatorname { m a x } \left\{d^{3}(S x, S y), d^{3}(A x, S x), d^{3}(S y, A y),\right.\right. \\
\left.\left.d^{2}(S y, A y), d(S x, A y), d(A x, S y)\right\}\right\}^{\lambda}
\end{gathered}
$$

for all $x, y \in X$, where $\lambda \in(0,1)$;

$\left(C_{7}\right) A$ is continuous;

$\left(C_{8}\right)$ the pair $A, S$ is semi-compatible.

Then $A$ and $S$ have a unique common fixed point in $X$.

Lemma 2.3. Let $A$ and $S$ be mappings of a multiplicative metric space $(X, d)$ into itself. Assume that $S$ is continuous. Then $A$ and $S$ are semicompatible if and only if $A$ and $S$ are compatible.

Proof. Consider a sequence $\left\{x_{n}\right\}$ in $X$ such that $\left\{A x_{n}\right\} \rightarrow u$ and $\left\{S x_{n}\right\} \rightarrow$ $u$. Since $S$ is continuous, we have $\left\{S A x_{n}\right\} \rightarrow S u$.

Suppose that $A$ and $S$ are semi-compatible. Then $\lim _{n \rightarrow \infty} d\left(A S x_{n}, S u\right)=1$ and $\lim _{n \rightarrow \infty} d\left(S A x_{n}, S u\right)=1$. Now

$$
d\left(A S x_{n}, S A x_{n}\right) \leq d\left(A S x_{n}, S u\right) \cdot d\left(S u, S A x_{n}\right) .
$$

By taking limit as $n \rightarrow \infty$, we get $\lim _{n \rightarrow \infty} d\left(S A x_{n}, A S x_{n}\right)=1$. Hence $A$ and $S$ are compatible.

Conversely, suppose that $A$ and $S$ are compatible. Then we have $\lim _{n \rightarrow \infty} d\left(A S x_{n}\right.$, $\left.S A x_{n}\right)=1$ and $\lim _{n \rightarrow \infty} d\left(S A x_{n}, S u\right)=1$. Now

$$
d\left(A S x_{n}, S u\right) \leq d\left(A S x_{n}, S A x_{n}\right) \cdot d\left(S A x_{n}, S u\right) .
$$

By taking limit as $n \rightarrow \infty$, we get $\lim _{n \rightarrow \infty} d\left(A S x_{n}, S u\right)=1$, that is, $A$ and $S$ are semi-compatible.

Theorem 2.4. Let $A, B, S$ and $T$ be mappings of a complete multiplicative metric spaces $(X, d)$ into itself satisfying

$$
\begin{gathered}
A^{a}(X) \subset T^{t}(X) \quad \text { and } \quad B^{b}(X) \subset S^{s}(X) \\
d\left(A^{a} x, B^{b} y\right) \leq\left\{\operatorname { m a x } \left\{d\left(S^{s} x, T^{t} y\right), d\left(S^{s} x, A^{a} x\right), d\left(T^{t} y, B^{b} y\right)\right.\right. \\
\left.\left.\left(d\left(S^{s} x, B^{b} y\right) \cdot d\left(A^{a} x, T^{t} y\right)\right)^{\frac{1}{2}}\right\}\right\}^{\lambda}
\end{gathered}
$$

for all $x, y \in X$, where $\lambda \in(0,1)$ and $a, b, s, t \in \mathbb{N}$.

$\left(C_{11}\right) S$ and $T$ are continuous;

$\left(C_{12}\right)$ the pairs $A, S$ and $B, T$ are semi-compatible.

Then $A, B, S$ and $T$ have a unique common fixed point in $X$. 
Proof. Let $x_{0} \in X$ be any arbitrary point. Since $A^{a}(X) \subset T^{t}(X)$ and $B^{b}(X) \subset S^{s}(X)$ there exists a point $x_{1} \in X$ such that $A^{a} x_{0}=T^{t} x_{1}$ and for this point $x_{1}$, there exists a point $x_{2} \in X$ such that $B^{b} x_{1}=S^{s} x_{2}$. Inductively, we can define a sequence $\left\{y_{n}\right\}$ in $X$ such that

$$
y_{2 n+1}=A^{a} x_{2 n}=T^{t} x_{2 n+1}, \quad y_{2 n}=B^{b} x_{2 n-1}=S^{s} x_{2 n}
$$

for $n=0,1,2, \ldots$.

Now on putting $x=x_{2 n}$ and $y=x_{2 n+1}$ in $\left(C_{10}\right)$, we have

$$
\begin{aligned}
d & \left(y_{2 n+1}, y_{2 n+2}\right) \\
= & d\left(A^{a} x_{2 n}, B^{b} x_{2 n+1}\right) \\
\leq & \left\{\operatorname { m a x } \left\{d\left(S^{s} x_{2 n}, T^{t} x_{2 n+1}\right), d\left(S^{s} x_{2 n}, A^{a} x_{2 n}\right), d\left(T^{t} x_{2 n+1}, B^{b} x_{2 n+1}\right),\right.\right. \\
& \left.\left.\left(d\left(S^{s} x_{2 n}, B^{b} x_{2 n+1}\right) \cdot d\left(A^{a} x_{2 n}, T^{t} x_{2 n+1}\right)\right)^{\frac{1}{2}}\right\}\right\}^{\lambda} \\
= & \left\{\operatorname { m a x } \left\{d\left(y_{2 n}, y_{2 n+1}\right), d\left(y_{2 n}, y_{2 n+1}\right), d\left(y_{2 n+1}, y_{2 n+2}\right),\right.\right. \\
& \left.\left.\left(d\left(y_{2 n}, y_{2 n+2}\right) \cdot d\left(y_{2 n+1}, y_{2 n+1}\right)\right)^{\frac{1}{2}}\right\}\right\}^{\lambda} \\
=\{ & \left.\max \left\{d\left(y_{2 n}, y_{2 n+1}\right), d\left(y_{2 n+1}, y_{2 n+2}\right),\left(d\left(y_{2 n}, y_{2 n+2}\right)\right)^{\frac{1}{2}}\right\}\right\}^{\lambda} \\
= & \left\{\operatorname { m a x } \left\{d\left(y_{2 n}, y_{2 n+1}\right), d\left(y_{2 n+1}, y_{2 n+2}\right),\right.\right. \\
& \left.\left.\left(d\left(y_{2 n}, y_{2 n+1}\right) \cdot d\left(y_{2 n+1}, y_{2 n+2}\right)\right)^{\frac{1}{2}}\right\}\right\}^{\lambda} .
\end{aligned}
$$

If $d\left(y_{2 n+1}, y_{2 n+2}\right) \geq d\left(y_{2 n}, y_{2 n+1}\right)$, then

$$
d\left(y_{2 n+1}, y_{2 n+2}\right) \leq d^{\lambda}\left(y_{2 n+1}, y_{2 n+2}\right),
$$

which is a contradiction. So,

$$
d\left(y_{2 n+1}, y_{2 n+2}\right) \leq d^{\lambda}\left(y_{2 n}, y_{2 n+1}\right) .
$$

Similarly, we have

$$
d\left(y_{2 n}, y_{2 n+1}\right) \leq d^{\lambda}\left(y_{2 n-1}, y_{2 n}\right)
$$

Hence for all $n$,

$$
d\left(y_{n}, y_{n+1}\right) \leq d^{\lambda}\left(y_{n}, y_{n-1}\right) .
$$

Continue like this, we get

$$
\begin{aligned}
d\left(y_{n+1}, y_{n}\right) & \leq d^{\lambda}\left(y_{n}, y_{n-1}\right) \\
& \leq d^{\lambda^{2}}\left(y_{n-1}, y_{n-2}\right) \\
& \leq \cdots \leq d^{\lambda^{n}}\left(y_{1}, y_{0}\right)
\end{aligned}
$$


Let $m, n \in \mathbb{N}$ with $m>n$.

$$
\begin{aligned}
d\left(y_{m}, y_{n}\right) & \leq d\left(y_{m}, y_{m-1}\right) \cdots d\left(y_{n+1}, y_{n}\right) \\
& \left.\leq d^{\lambda^{m-1}}\left(y_{1}, y_{0}\right)\right)+\cdots+d^{\lambda^{n}}\left(y_{1}, y_{0}\right) \\
& \leq d^{\frac{\lambda^{n}}{1-\lambda}}\left(y_{1}, y_{0}\right) .
\end{aligned}
$$

This implies that $d\left(y_{m}, y_{n}\right) \rightarrow 1$ as $m, n \rightarrow \infty$. Hence $\left\{y_{n}\right\}$ is a multiplicative Cauchy sequence. Therefore $y_{n} \rightarrow z \in X(n \rightarrow \infty)$. Thus $A^{a} x_{2 n}, T^{t} x_{2 n+1}$, $B^{b} x_{2 n+1}$ and $S^{s} x_{2 n} \rightarrow u \in X$.

Since $S$ is continuous, $\lim _{n \rightarrow \infty} S^{s} A^{a} x_{2 n}=S^{s} u$ and $\lim _{n \rightarrow \infty} S^{s} S^{s} x_{2 n}=S^{s}(u)$. Also since $T$ is continuous, $\lim _{n \rightarrow \infty} T^{t} B^{b} x_{2 n+1}=T^{t} u$ and $\lim _{n \rightarrow \infty} T^{t} T^{t} x_{2 n+1}=T^{t} u$.

Further since the pairs $A, S$ and $B, T$ are semi-compatible, by Lemma 2.3, the pairs $A, S$ and $B, T$ are compatible. It follows easily that the pairs $A^{a}, S^{s}$ and $B^{b}, T^{t}$ are compatible for all $a, b, s, t \in \mathbb{N}$. Hence we have $\lim _{n \rightarrow \infty} d\left(A^{a} S^{s} x_{2 n}\right.$, $\left.S^{s} A^{a} x_{2 n}\right)=1$ and hence $\lim _{n \rightarrow \infty} A^{a} S^{s} x_{2 n}=S^{s} u$. Also, we have $\lim _{n \rightarrow \infty} B^{b} T^{t} x_{2 n+1}=$ $T^{t} u$.

On putting $x=S^{s} x_{2 n}$ and $y=T^{t} x_{2 n+1}$ in $\left(C_{10}\right)$, we have

$$
\begin{aligned}
& d\left(A^{a} S^{s} x_{2 n}, B^{b} T^{t} x_{2 n+1}\right) \\
& \leq\left\{\operatorname { m a x } \left\{d\left(S^{s} S^{s} x_{2 n}, T^{t} T^{t} x_{2 n+1}\right), d\left(S^{s} S^{s} x_{2 n}, A^{a} S^{s} x_{2 n}\right),\right.\right. \\
& \quad d\left(T^{t} T^{t} x_{2 n+1}, B^{b} T^{t} x_{2 n+1}\right), \\
& \left.\left.\quad\left(d\left(S^{s} S^{s} x_{2 n}, B^{b} T^{t} x_{2 n+1}\right) \cdot d\left(A^{a} S^{s} x_{2 n}, T^{t} T^{t} x_{2 n+1}\right)\right)^{\frac{1}{2}}\right\}\right\}^{\lambda} .
\end{aligned}
$$

By taking $n \rightarrow \infty$, we get

$$
\left.d\left(S^{s} u, T^{t} u\right) \leq d^{\lambda}\left(S^{s} u, T^{t} u\right)\right)
$$

which gives that $S^{s} u=T^{t} u$.

Similarly, one can find that $A^{a} u=B^{b} u$ and $A^{a} u=T^{t} u$. Therefore, $A^{a} u=$ $B^{b} u=S^{s} u=T^{t} u$.

Now we shall prove $A^{a} u=u$. Putting $x=u$ and $y=x_{2 n+1}$ in $\left(C_{10}\right)$, we have

$$
\begin{aligned}
& d\left(A^{a} u, B^{b} x_{2 n+1}\right) \\
& \leq\left\{\operatorname { m a x } \left\{d\left(S^{s} u, T^{t} x_{2 n+1}\right), d\left(S^{s} u, A^{a} u\right), d\left(T^{t} x_{2 n+1}, B^{b} x_{2 n+1}\right),\right.\right. \\
& \left.\quad\left(d\left(S^{s} u, B^{b} x_{2 n+1}\right) \cdot d\left(A^{a} u, T^{t} x_{2 n+1}\right)\right)^{\frac{1}{2}}\right\}^{\lambda} .
\end{aligned}
$$

By taking $n \rightarrow \infty$, we get $A^{a} u=u$. Hence $u$ is common fixed point of $A^{a}, B^{b}$, $S^{s}$ and $T^{t}$. 
Next in order to prove the uniqueness of $z$, let $z(\neq u)$ be another common fixed point of $A^{a}, B^{b}, S^{s}$ and $T^{t}$.

On putting $x=u$ and $y=z$ in $\left(C_{10}\right)$, we have

$$
\begin{aligned}
d(u, z)= & d\left(A^{a} u, B^{b} z\right) \\
\leq & \left\{\operatorname { m a x } \left\{d\left(S^{s} u, T^{t} z\right), d\left(S^{s} u, A^{a} u\right), d\left(T^{t} z, B^{b} z\right),\right.\right. \\
& \left.\left(d\left(S^{s} u, B^{b} z\right) \cdot d\left(A^{a} u, T^{t} z\right)\right)^{\frac{1}{2}}\right\}^{\lambda} \\
= & \left\{\max \left\{d(u, z), d(u, u), d(z, z),(d(u, z) \cdot d(u, z))^{\frac{1}{2}}\right\}^{\lambda}\right. \\
= & \{\max \{d(u, z), 1,1, d(u, z)\}\}^{\lambda} \\
= & d^{\lambda}(u, z),
\end{aligned}
$$

which implies that $u=z$. Hence $u$ is a unique common fixed point of $A^{a}, B^{b}$, $S^{s}$ and $T^{t}$.

Finally we prove that this point $u$ is common fixed point of $A, B, S$ and $T$. Now $A u=A\left(A^{a} u\right)=A^{a}(A u)$ and $A u=A\left(S^{s} u\right)=S^{s}(A u)$ from $\left(C_{14}\right)$. Hence $A u$ is a common fixed point of $A^{a}$ and $S^{s}$.

Also, we have $B u=B\left(B^{b} u\right)=B^{b}(B u)$ and $B u=B\left(T^{t} u\right)=T^{t}(B u)$ from $\left(C_{14}\right)$. Hence $B u$ is a common fixed point of $B^{b}$ and $T^{t}$.

Now on putting $x=A u$ and $y=B u$ in $\left(C_{10}\right)$, we have

$$
\begin{aligned}
d(A u, B u)= & d\left(A^{a} A u, B^{b} B u\right) \\
\leq & \left\{\operatorname { m a x } \left\{d\left(S^{s} A u, T^{t} B u\right), d\left(S^{s} A u, A^{a} u\right), d\left(T^{t} B u, B^{b} B u\right),\right.\right. \\
& \left.\left(d\left(S^{s} A u, B^{b} B u\right) \cdot d\left(A^{a} A u, T^{t} B u\right)\right)^{\frac{1}{2}}\right\}^{\lambda} \\
= & d^{\lambda}(A u, B u),
\end{aligned}
$$

which implies that $A u=B u$.

Also, now on putting $x=S u$ and $y=T u$ in $\left(C_{10}\right)$, we have

$$
\begin{aligned}
d(S u, T u)= & d\left(A^{a} S u, B^{b} T u\right) \\
\leq & \left\{\operatorname { m a x } \left\{d\left(S^{s} S u, T^{t} T u\right), d\left(S^{s} S u, A^{a} u\right), d\left(T^{t} T u, B^{b} T u\right),\right.\right. \\
& \left.\left.\left(d\left(S^{s} S u, B^{b} T u\right) \cdot d\left(A^{a} S u, T^{t} T u\right)\right)^{\frac{1}{2}}\right\}\right\}^{\lambda} \\
= & d^{\lambda}(S u, T u),
\end{aligned}
$$

which implies that $S u=T u$. Since $u$ is a unique common fixed point of $A^{a}, B^{b}$, $S^{s}$ and $T^{t}$, we have $A u=B u$ is a common fixed point of $A^{a}, S^{s}$ and $S u=T u$ is a common fixed point of $B^{b}, T^{t}$. Thus $u=A u=B u=S u=T u$. Therefore $u$ is a unique common fixed point of $A, B, S$ and $T$. 


\section{References}

[1] M. Abbas, B. Ali, Y.I. Suleiman, Common fixed points of locally contractive mappings in multiplicative metric spaces with application, Int. J. Math. Math. Sci., 2015 (2015), Article ID 218683, 7 pages. doi:10.1155/2015/218683.

[2] M.A. Ahmed, Common fixed point theorems for weakly compatible mappings, Rocky Mountain J. Math., 33 (2003), 1189-1203.

[3] A.E. Bashirov, E.M. Kurplnara, A. Ozyapici, Multiplicative calculus and its applications, J. Math. Anal. Appl., 337 (2008), 36-48. doi: 10.1016/j.jmaa.2007.03.081

[4] Y.J. Cho, B.K. Sharma, D.R. Sahu, Semi-compatibility and fixed points, Math. Japon., 42 (1995), 91-98.

[5] R. Chugh, S. Kumar, Common fixed points for weakly compatible maps, Proc. Indian Acad. Sci. Math. Sci., 111 (2001), 241-247.

[6] Lj.B. Ćirić, J.S. Ume, Some common fixed point theorems for weakly compatible mappings, J. Math. Anal. Appl., 314 (2006), 488-499. doi: 10.1016/j.jmaa.2005.04.007

[7] X. He, M. Song, D. Chen, Common fixed points for weak commutative mappings on a multiplicative metric space, Fixed Point Theory Appl., 48 (2014), 9 pages. doi: 10.1186/1687-1812-2014-48

[8] G. Jungck, Common fixed points for noncontinuous nonself maps on nonmetric spaces, Far East J. Math. Sci., 4 (1996), 199-215.

[9] S. Kang, P. Kumar, S. Kumar, P. Nagpal, S.K Garg, Common fixed points for compatible mappings and its variants in multiplicative metric spaces, Int. J. Pure Appl. Math., 102 (2015), 383-406. doi: 10.12732/ijpam.v102i2.14

[10] M. Özavsar, A.C. Çevikel, Fixed points of multiplicative contraction mappings on multiplicative metric spaces, arXiv:1205.5131v1 [math.GM], 2012.

[11] V. Popa, A general fixed point theorem for four weakly compatible mappings satisfying an implicit relation, Filomat, 19 (2005), 45-51.

[12] M. Sarwar, R. Badshah-e, Some unique fixed point theorems in multiplicative metric space, arXiv:1410.3384v2 [math.GM], 2014. 